V O R W O R T.

Salve duleis patria

Suavis Sucvorum Suevia:

Wohl werden auch Sie, hochverehrter Freund, wenn Sie die geschichte unserer mundart entgegennehmen wollen, gerne des schönen landes und treuen volkes gedenken, in dessen mitte Sie kurze aber fruchtbare jahre gewirkt haben. Mit dem gruss an die heimat verknüpft sich mir so die erinnerung an die Tübinger lehrzeit, die erinnerung an jene im leben des einzelnen so wichtigen augenblicke, wo mit einem schlage die auffassung der thatsachen sich verändert und der sichere hort des wissens sich zu sammeln beginnt. Die allgemeinen kategorien der denkthätigkeit lassen sich bekanntermassen nicht wie das a-b-c an den fingern herzählen, sie sind in steter entwicklung; der fortschritt des individuums wie der wissenschaft kommt nicht sowohl in ihrer erfassung als vielmehr wesentlich in der klarheit über die einordnung der vorstellungen in die verschiedenen kategorien zum ausdruck. So genügt es also keineswegs dem ruf der masse nach konstatirung der erfahrungsthatsachen zu gehorchen, so lange das ergebniss neuer beobachtung nicht allseitig in wechselbeziehung zum bereits erworbenen gesetzt wird, kann von wissenschaftlicher leistung nicht die rede sein. In diesem sinne ist die folgende darstellung gedacht. Ich habe es mir angelegen 
sein lassen, unbekanntes oder versäumtes material zur veranschaulichung meiner behauptungen herbeizuschaffen, ich habe mich bemüht, jede einzelnheit erfahrungsgemäss sicher zu stellen und in den richtigen zusammenhang einzuordnen: bei der arbeit ist mir mehr und mehr jede einzelne form ein symbol des gesammtlebens geworden. So möchte ich meine geschichte der schwäbischen mundart gerne als beitrag zur historischen anthropologie Schwabens betrachtet wissen.

Die sprache mit den äusserungen ihrer lebensformen, von wortschöpfung, wortbildung, wortschatz u. s. w. ganz abgesehen, ist eine ausserordentlich ergiebige quelle für die erkenntniss der untergegangenen menschheit. Durch die psychologische begründung des sprachlebens, wie sie uns von Hermann Paul gegeben worden ist, sind uns unsere ahnen viel vertrauter, ihr seelenleben ansprechender geworden. Wie der kreislauf des blutes auch durch ihre körper seinen gesetzmässigen weg gegangen, so hat der luftstrom in ihren schallbildenden organen sich zum sprachlaute gebrochen, so hat die sprechthätigkeit ihre phantasie angeregt und so haben auch ihre anschauungen und vorstellungen in der mechanik des selbstbewusstseins ihr wundersames spiel getrieben („analogiebildung ") und das dunkle rätsel der.umsetzung des gedankens in die schallbewegung des lautes hat auch in den vergangenen jahrhunderten bestanden. Die übereinstimmung der elementaren lebenskräfte ist für den geschichtschreiber nicht bloss methodologisch fruchtbar.

Nach dem bilde, das meine darstellung von der entwicklungsgeschichte des schwäbischen lautbestandes gibt, werden die ansichten der principienwissenschaft über die allgemeinen faktoren des lautwandels wesentlich zu modificiren sein. Paul sieht die eigentliche ursache der veränderung in der gewöhnlichen sprechthätigkeit, bei welcher dem einzelnen immer ein bestimmtes mass individueller freiheit bleibe. Die bethätigung dieser individuellen freiheit wirke zurück auf den psychischen organismus des sprechenden, zugleich aber auch auf den organismus des 
hörenden. Durch die summirung einer reihe minimalster verschiebungen in den einzelnen organismen ergibt sich dann als gesammtresultat die verschiebung der usuellen lautbildung. Diese verschiebung könne aber nicht wohl zu stande kommen, ohne dass das individuum beeinflussung von andern individuen erfahre. Die hauptperiode dieser beeinflussung sei die zeit der spracherlernung im kindlichen alter, die vorgänge bei der spracherlernung seien die wichtigsten ursachen für die sprachveränderung. Der akt der sprachübertragung von einer generation auf die andere, nächstfolgende, wird für die lautverschiebung verantwortlich gemacht. Die consequenz ist eine immerwährende ununterbrochene kleine und kleinste veränderung in der erzeugung der mundartlichen laute; nach generationen summiren sich diese kleinsten grössen zu einer akustisch und psychisch fassbaren umwandlung. Eine solche auffassung ist fremdartig, schon deswegen, weil sie die einzelnen vorgänge isolirt und die lauterzeugung zu sehr in das interesse des individuums rückt. Die lauterzeugung ist ein psychophysischer vorgang. Sie ist allein abhängig von der function der sprachorgane und deren wechselbeziehung zum bewusstsein. Übereinstimmung der lauterzeugung oder mit andern worten eine in sich überstimmende bei allen angehörigen gleichmässige mundart ist nur denkbar bei identischem bau und identischer function der organe. Da nun aber so viel wir wissen, die sprachorgane des homo sapiens auf der ganzen erde ein und dieselben sind, kann die verschiedenheit der sprachen nur auf verschiedenheit der function derselben beruhen, kurz die verschiedenheit der muskel- und nerventhätigkeit involvirt die unterschiede der mundarten nach ihrer rein lautlichen seite. Wir können nur behaupten, identische lauterzeugung hänge von identischer function der die schallbildenden und schallmodificirenden körper beherrschenden organe (muskeln) ab. Soweit die identität der muskelfunction reicht, ziehen wir die grenzen einer mundart, eine lautveränderung ist nur denkbar, wenn in den functionsorganen eine veränderung 
sein lassen, unbekanntes oder versäumtes material zur veranschaulichung meiner behauptungen herbeizuschaffen, ich habe mich bemüht, jede einzelnheit erfahrungsgemäss sicher zu stellen und in den richtigen zusammenhang einzuordnen: bei der arbeit ist mir mehr und mehr jede einzelne form ein symbol des gesammtlebens geworden. So möchte ich meine geschichte der schwäbischen mundart gerne als beitrag zur historischen anthropologie Schwabens betrachtet wissen.

Die sprache mit den äusserungen ihrer lebensformen, von wortschöpfung, wortbildung, wortschatz u. s. w. ganz abgesehen, ist eine ausserordentlich ergiebige quelle für die erkenntniss der untergegangenen menschheit. Durch die psychologische begründung des sprachlebens, wie sie uns von Hermann Paul gegeben worden ist, sind uns unsere ahnen viel vertrauter, ihr seelenleben ansprechender geworden. Wie der kreislauf des blutes auch durch ihre körper seinen gesetzmässigen weg gegangen, so hat der luftstrom in ihren schallbildenden organen sich zum sprachlaute gebrochen, so hat die sprechthätigkeit ihre phantasie angeregt und so haben auch ihre anschauungen und vorstellungen in der mechanik des selbstbewusstseins ihr wundersames spiel getrieben („analogiebildung") und das dunkle rätsel der.umsetzung des gedankens in die schallbewegung des lautes hat auch in den vergangenen jahrhunderten bestanden. Die übereinstimmung der elementaren lebenskräfte ist für den geschichtschreiber nicht bloss methodologisch fruchtbar.

Nach dem bilde, das meine darstellung von der entwicklungsgeschichte des schwäbischen lautbestandes gibt, werden die ansichten der principienwissenschaft über die allgemeinen faktoren des lautwandels wesentlich zu modificiren sein. Paul sieht die eigentliche ursache der veränderung in der gewöhnlichen sprechthätigkeit, bei welcher dem einzelnen immer ein bestimmtes mass individueller freiheit bleibe. Die bethätigung dieser individuellen freiheit wirke zurück auf den psychischen organismus des sprechenden, zugleich aber auch auf den organismus des 
hörenden. Durch die summirung einer reihe minimalster verschiebungen in den einzelnen organismen ergibt sich dann als gesammtresultat die verschiebung der usuellen lautbildung. Diese verschiebung könne aber nicht wohl zu stande kommen, ohne dass das individuum beeinflussung von andern individuen erfahre. Die hauptperiode dieser beeinflussung sei die zeit der spracherlernung im kindlichen alter, die vorgänge bei der spracherlernung seien die wichtigsten ursachen für die sprachveränderung. Der akt der sprachübertragung von einer generation auf die andere, nächstfolgende, wird für die lautverschiebung verantwortlich gemacht. Die consequenz ist eine immerwährende ununterbrochene kleine und kleinste veränderung in der erzeugung der mundartlichen laute; nach generationen summiren sich diese kleinsten grössen zu einer akustisch und psychisch fassbaren umwandlung. Eine solche auffassung ist fremdartig, schon deswegen, weil sie die einzelnen vorgänge isolirt und die lauterzeugung zu sehr in das interesse des individuums rückt. Die lauterzeugung ist ein psychophysischer vorgang. Sie ist allein abhängig von der function der sprachorgane und deren wechselbeziehung zum bewusstsein. Übereinstimmung der lauterzeugung oder mit andern worten eine in sich überstimmende bei allen angehörigen gleichmässige mundart ist nur denkbar bei ide ntischem bau und identischer function der organe. Da nun aber so viel wir wissen, die sprachorgane des homo sapiens auf der ganzen erde ein und dieselben sind, kann die verschiedenheit der sprachen nur auf verschiedenheit der function derselben beruhen, kurz die verschiedenheit der muskel-und nerventhätigkeit involvirt die unterschiede der mundarten nach ihrer rein lautlichen seite. Wir können nur behaupten, identische lauterzeugung hänge von identischer function der die schallbildenden und schallmodificirenden körper beherrschenden organe (muskeln) ab. Soweit die identität der muskelfunction reicht, ziehen wir die grenzen einer mundart, eine lautveränderung ist nur denkbar, wenn in den functionsorganen eine veränderung 
eintritt. Weiter darf meiner meinung nach die vorsichtige abstracte deduction nicht gehen, die beobachtung des thatsächlichen sprachlebens hat das letzte wort. Fernere bestätigung oder widerlegung erwartend, wage ich die auf meinem arbeitsfeld gewonnenen resultate auszubeuten, in der hoffinung, damit auf andern dialectgebieten die beobachtung anzuregen.

Ich habe $\$ \S 141.193$ in chronologischen tabellen zusammengestellt, wie sich die (nach der schriftlichen überlieferung) vollzogenen lautveränderungen auf die jahrhunderte verteilen. Die betr. zahlen kann ich samt und sonders nicht als der datirung der thatsächlichen ereignisse entsprechend halten, sondern glaube nicht bloss, dass die ansätze um grössere zeiträume zu spät, sondern auch, dass die verschiedenen lautveränderungen einander noch viel näher zu rücken sind, so dass in einer reihe von jahrhunderten eine allmähliche aber radicale umwandlung der lauterzeugung sich vollzogen hätte, die sowohl für consonantismus als vocalismus eine verschiebung der articulationsstellen und articulationsarten mit sich gebracht. Die ursache hiefür haben wir nach dem vorhin bemerkten in einer veränderung der muskel- (und nerven-?) function zu suchen. Einzelne muskeln, vormals wahrscheinlich mit strafferer energie thätig, haben an intensität der spannung verloren, andere gewonnen, muskelstränge, die vordem in activität gewesen, sind ausser dienst gesetzt und haben ihre function an andere abgegeben, folglich sind die passiven organe wie kehlkopf, zunge, kiefer und lippen anders dirigirt worden. In solchen vorgängen spielt sich die lautgeschichte $a b$, sie bringt weder fortschritt noch verfall, naturgesetze können durch culturbegriffe nicht einmal erläutert, geschweige definirt werden. Nun geht aus $\$ \$ 141.193$ hervor, dass seit dem 14. jh. überhaupt keine veränderung der lautbildung nachgewiesen werden kann, mit andern worten, dass seit 5 jahrhunderten der schwäbische lautstand sich überhaupt nicht mehr verändert hat; und ich bezweifle nicht, dass die stabilität desselben in noch ältere zeiten zurückreicht. Dieses merkwürdige er- 
eigniss der sprachveränderung darf nun aber nicht isolirt gehalten, sondern muss in den zusammenhang der stammesgeschichte gestellt werden. Wir wissen, dass der Schwabenstamm im dritten jahrhundert aus seinen nördlichen wohnsitzen in die Neckargegenden eingewandert ist. Was liegt näher als mit dieser veränderung des wohnsitzes die veränderung der mundart $\mathrm{zu}$ combiniren? So meine ich denn und wage zu behaupten: unter dem veränderten himmel, bei verändertem luftdruck, unter gänzlich anderen boden- und lebensverhältnissen hat sich (in Darwin'schem sinne) die physikalische function der sprachorgane den neuen verhältnissen im lauf der jahrhunderte angepasst, soweit, in strengster auffassung, identische function der betr. organe vordem vorhanden gewesen, soweit dieselben äusseren factoren gewirkt haben, hat sich dann auch dieselbe mundart von der nachbarschaft abgesondert. Ins detail diese hypothese $\mathrm{zu}$ verfolgen, ist nicht möglich, für mein begrenztes wissen steht derselben nichts im wege und ich betrachte sie vorerst als eine schöne bestätigung der weltansicht von der entstehung der arten (mundart, stamm). So lange die äusseren bedingungen für unser volksleben dieselben sind und bleiben ist nicht einzusehen, dass die mundart sich verändern, die function der ausübenden organe wechseln könnte. Die ansicht von der fortwährenden verschiebung der lautbildung bei der übertragung der lautform von generation zu generation hat vorerst die erfahrung gegen sich. Möchte doch meine darlegung der lautveränderung und ihrer ursachen recht eindringlich an weiteren mundarten geprüft werden.

Sicher ist jedenfalls auch für den strengsten schriftgläubigen, dass seit jahrhunderten die articulationsstellen eingenommen sind, welche die mundart heute zeigt, meine darstellung bringt hiefür mathematisch zuverlässige zeugnisse in menge. Fraglich könnte nur noch sein, ob auch die articulationsart von heute seit jahrhunderten existirt, die zweifel können aber schon deswegen nicht béstehen, weil ich gerade die innersten merkmale der articulationsarten (wie verlust des stimmtons, accent, melodie) im 
zusammenhang uralter veränderungen habe feststellen können. Im ganzen ist der nachweis des alters der mundart wohl der erste im zusammenhang gegebene, doch vgl. Germ. 25,117. Noreen, Grundriss der germ. philologie I, 431. Weinhold, Die deutschen in Schlesien s. 214, von den anschauungen Adelungs Wörterb. d. hd. mundart. s. VI. VIII. Magazin II, 2, 32 nicht zu reden.

Die mechanische erklärung der lautverschiebung schliesst in sich eine mechanische deutung der lautgesetze. In der verständigung über diesen begriff hat es seitdem immer an ausreichendem, thatsächlich beobachtetem material gefehlt. Namentlich ist durch einen schon in sich unmöglichen kosmopolitischen standpunkt verwirrung gestiftet worden. Ich lege jetzt in meiner darstellung eine reihe von sog. lautgesetzen vor, die für jeden unbefangenen klarheit in das problem bringen werden. Die schwäbische nasalirung ist ein vorgang, der sämtliche in betracht kommende fälle betroffen hat, ein lautgesetz, für welches keine einzige ausnahme beizubringen ist, in allen (bekannten) fällen ist, wie ich annehme im 12. jh., in der verbindung von vocal und nasal nasenresonanz des vocals eingetreten. Es ist $\mathrm{mir}$ auch gelungen $\S 16$ dieses gesetzes in seinen folgen auf die ursache zurückzuführen. Wenn ich mit der annahme recht habe, dass die nasalirung der vocale auf einer historisch eingetretenen contraction des musculus glossopalatinus beruht, kann vernünftiger weise kein mensch an der ausnahmslosigkeit des gesetzes und seiner mechanischen deutung zweifeln. Genau dasselbe gilt von dem wegfall der lippenrundung bei $\ddot{o}$ und ii $(\S 140,1)$, von dem quantitäts- (\$122) und dem assimilationsgesetz ( $\S 192$ ), die beiden letzteren aufs engste zusammengehörig. Aber wenn in diesen beiden fällen die historischen belege für die gesetzmässigkeit stark decimirt sind, lässt sich an ihnen besonders instructiv lernen, dass im sprachleben nicht bloss mechanische kraft und wirkung sondern auch psychische bewegungen sich damit zu unlöslicher einheit verbinden. Vermöge des mechanischen vorgangs der muskelbewegung constituirt sich ein lautgesetz aus allen 
lautformen, bei deren erzeugung die betr. muskelbewegung beteiligt ist. Diese beteiligung setzt aber vollständige identität sämmtlicher sprachelemente voraus, wo diese identität nicht vorhanden, darf von vornherein der eintritt des lautgesetzes überhaupt nicht erwartet werden. So entstehen vielfach, selbst bei einem und demselben wort nicht bloss sogenannte satzdoubletten, sondern eine vielleicht unendliche reihe verschiedener formen, durch deren existenz der statistische nachweis des lautgesetzes insofern gefährdet ist, als durch die concurrenz des bewusstseins und der gedächtnisskraft nicht alle formen gleich lebenskräftig sind. Die sog. analogiebildung besagt folglich nichts anderes, als dass im verlauf der sprechthätigkeit die menge des gedächtnissstoffes reducirt und ohne erkennbare regel bald die eine bald die andere form von dem günstigen loos der erhaltung betroffen wird. Die mechanische gesetzmässigkeit der lautbildung und lautveränderung (lautgesetz) kann nicht streng genug von den sekundären bewusstseinsfunctionen geschieden werden, aber einem durch innere und äussere gründe gewährleisteten lautgesetz seinen gesetzmässig mechanischen charakter bestreiten $\mathrm{zu}$ wollen, weil eine bald grössere bald geringere masse von wortformen dagegen zu sprechen scheint, nur weil man die verschiedenheit der bedingungen nicht bedacht hat, heisst die grundlagen des sprachlebens verkennen.

Die sog. hd. lautverschiebung möge zur illustration dieser ansichten beigezogen werden. Im voraus sei bemerkt, dass dieser process nur provinciell verfolgt werden kann, dass jede einzelne mundart denselben selbständig und eigenartig durchgemacht hat. Es trägt durchaus nicht zur klarheit bei, wenn man sich hierzu des bildes von der wellenbewegung bedient. Um zum verständniss dieser erscheinung zu gelangen, wäre in erster linie erforderlich, die constitutiven sprachfactoren derjenigen periode $\mathrm{zu}$ kennen, die als mutterschoos der folgenden $\mathrm{zu}$ betrachten ist. Zweitens ist es unzulässig, wo es sich um die erklärung handelt, die verschiebung einer geringen zahl von consonanten $\mathrm{zu}$ isoliren oder gar jeden einzelnen consonanten für sich 
zu betrachten. Schon die gleichzeitigkeit der belege z. b. für die ahd. monophthongirung und anderer vocalischer erscheinungen sollte daran gemahnt haben, dass in jenen grauen zeiten veränderungen über die hd. stämme hingegangen sind, deren zusammenhang nicht auf ein paar consonanten eingeschrumpft werden darf. Methodologisch empfiehlt es sich daher, den bereich eines sog. lautgesetzes von vornherein möglichst umfassend zu nehmen (z. b. assimilation $\S 126$. $§ 194)$, und die schicksale einzelner laute auf die triebkraft einer wurzel zurückzuführen, deren säfte sich mannigfach am lebensbaum der sprache verzweigen. Die veränderung in der activität $\mathrm{z}$. b. der zungenmuskulatur involviert nicht bloss eine verschiebung in der articulation der consonanten sondern auch der vocale. Beim heutigen stand unseres wissens ist es völlig ausgeschlossen, dass wir in die geheimnisse der phonetischen processe eindringen, welche zur radicalen umwälzung unserer hd. muttersprache geführt haben, aber es kann uns vorerst auch genügen, den weg, der zum ziele führt, in der perspective zu haben. Besonders wichtig scheint nun aber schliesslich, was ich $\S 168$ über accessorische wirkungen (sandhierscheinungen; $p f$ - aus $f$-) behauptet habe. Es bedarf also vielfach eindringender voruntersuchungen, dass wir gewisse elemente erst abziehen müssen, um die producte zu erhalten, die als directe schösslinge des lautgesetzes zu betrachten sind.

Ausser diesen hypothetischen erörterungen, die ich als unwillkürliche reflexbilder meiner arbeit gebe, habe ich wenig hinzuzufügen. In der behandlung des quellenmaterials war es besonders interessant in den ältesten denkmälern, den glossensammlungen des 10.-12. jahrhunderts zu verfolgen, wie sich verschiedene schichten sprachlicher ablagerungen über einander geschoben haben. Die chronologische sonde wird, auf weiteren gebieten verfolgt, viel zur klarheit in der auffassung der überlieferten ahd. sprachformen beitragen. Die auswahl des handschriftlichen materials ist eine zufällige. Ich habe ausgenützt, was mir auf der Stuttgarter öffentlichen bibliothek an sicheren 
schwäbischen texten des mittelalters zur hand war; manche verdienten eingehendere besprechung, zu der ich hoffentlich bald musse finde. Die urkunden des Stuttgarter Staatsarchivs habe ich, wie man meinen könnte, stiefmütterlich behandelt - die rechtfertigung wird meine darstellung selbst bringen. Die zusammenhängenden denkmäler localer herkunft liefern weit wertvolleres material als die früh in der formel erstarrten schriftstücke der kanzleien, eine erfahrung, die sich seit einiger zeit auch bezüglich anderer probleme geltend gemacht hat. Dass ich mich stets mit peinlicher strenge an die überlieferten formen gehalten, braucht nicht erst bemerkt zu werden; bezüglich der urkunden war für mich Paul, Germ. XX, 86 massgebend.

An neueren dialectformen habe ich gesammelt, was ich erreichen konnte, und gebe mich der hoffnung hin, alle lautschattirungen der schwäbischen mundart sei es an diesem oder jenem orte verzeichnet $z u$ haben. Einzelne unebenheiten der darstellung, die vielfach sehr schwierig gewesen ist, bitte ich zu entschuldigen.

Was ich über flexionserscheinungen und thatsachen der wortbildung gesammelt habe, ist in die lautstatistik verwoben, ich glaube nicht, dass ich eine wissenswerte thatsache aus diesen gebieten versäumt habe. In diesem sinne habe ich mir erlaubt meine darstellung als geschichte der schwäbischen mundart zu bezeichnen, wenn ich auch mundart vorwiegend in dem specifischen sinne des wortes genommen habe und sehr viel mehr darunter verstehe als mein buch bringt. Soweit flexionslehre und wortbildung in das gebiet der syntax fallen, bin ich an ihnen vorübergegangen, weil es für die probleme des satzbaues und der satzfügung an den grundlegenden principien der geschichtlichen entwicklung fehlt, auf die mein augenmerk stets gerichtet war. Ich kann aber auch nicht verschweigen, dass, so viele texte ich gelesen habe, es mir unmöglich wäre, ein einziges datum z. b. für den verlust des einfachen präteritums beizubringen.

Unter herzlichem dank an E. Sievers, dessen umsichtige sorgfalt ich bei der correctur zur seite haben 
durfte, sowie an die beamten der kgl. öffentlichen bibliothek und meinen stets willigen freund, herrn Archivassessor Dr. E. Schneider in Stuttgart, schliesse ich mit dem dringenden wunsch, dass von seiten der fachgenossen auf andern dialectgebieten ähnliche studien zur geschichte unserer muttersprache angeregt und unterstützt werden möchten.

Marburg i. H., November 1889.

\section{Friedrich Kanffmann.}

